


\title{
Real-time Registration of Paper Watermarks
}

\author{
Paul F Whelan \\ Vision Systems Laboratory \\ School of Electronic Engineering \\ Dublin City University \\ Dublin 9, Ireland \\ Ph: +353 17045489 \\ Fax: +353 17045508 \\ paul.whelan@eeng.dcu.ie \\ Pierre Soille ${ }^{1}$ \\ Silsoe Research Institute \\ Wrest Park, Silsoe, Bedfordshire \\ MK45 4HS, England \\ Ph.: + 44 (0)1525 860000 \\ Fax: +44 (0)1525 861735 \\ Pierre.Soille@bbsrc.ac.uk \\ Alexandru Drimbarean \\ Vision Systems Laboratory \\ School of Electronic Engineering \\ Dublin City University \\ Dublin 9, Ireland \\ Ph: +353 17045869 \\ Fax: +353 17045508 \\ drimbare@eeng.dcu.ie
}

April 24, 2002 
${ }^{1}$ Pierre Soille is now with the EC Joint Research Centre, Space Applications Institute, TP 441, I-21020 Ispra, Italy Email: Pierre.Soille@jrc.it 


\section{Abstract \\ Real-time Registration of Paper Watermarks}

The aim of this article is to outline the issues involved in the application of machine vision to the automatic extraction and registration of watermarks from continuous web paper. The correct identification and localisation of watermarks are key issues in paper manufacturing. As well as requiring the position of the watermark for defect detection and classification, it is necessary to insure its position on the paper prior to the cutting process.

Two paper types are discussed, with and without laid and chain lines (these lines appear as a complex periodic background to the watermark and further complicate the segmentation process). We will examine both morphological and Fourier approaches to the watermark segmentation process, concentrating specifically on those images with complex backgrounds. Finally we detail a system design suitable for real-time implementation.

Keywords: Watermark, Paper, Machine Vision, Morphology, Fourier. 


\section{Introduction}

Watermarks are generally used for security and quality purposes. They take the form of brand names, brook bonds or logos (Figure 1). They are typically formed by a pressing process using a metal stamp containing an impression of the desired pattern. This pattern induces a variation in the paper thickness and is implemented at the end of the wet stage of the paper production process. Currently, the watermark is captured on-line and displayed on a CRT beside an image of a good sample, where operators examine it occasionally. This enables the identification of gross errors, whereas the operators classify other defects on a subjective basis. Our aim is to automate the watermark registration as the first phase in the defect detection and classification process. Key to the success of this operation is the reliable segmentation of the watermark for plain and non-plain paper types. Non-plain paper samples contain laid and chain lines (Figure 1b). The horizontal laid lines are due to the wire mesh that is used to hold the paper during fabrication. The vertical chain lines are due to the thicker wire that is used to support the laid lines. The localisation of the watermark is also critical to the paper cutting operation, since some manufacturers require the watermark to appear in the same location on each cut sheet.

Previous research on watermark analysis has generally concentrated on 
techniques to determine authenticity and origin of watermarks on older historical documents. Techniques for capturing digital images of historical watermarks, using both transmitted and reflected light so as to discriminate watermarks from ink, are detailed in [1]. The extraction of watermarks on old hand-written documents is detailed in [2]. Finally, the management of a multimedia database of historical papers representing ancient watermarks is detailed in [3].

Our article is organised as follows. Section 2 details the image acquisition and the resulting image characteristics. Section 3 outlines our approach for processing watermarks on plain paper. More sophisticated techniques allowing the extraction of watermarks on non-plain paper are proposed in Section 4. A design outline for a real-time implementation is discussed in Section 5. Before concluding, tests and results are presented in Section 6.

\section{Production Environment}

The production development system (Figure 2) used for this project consisted of the following elements:

- A high frequency aperture fluorescent backlighting configuration along with an acrylic rod to focus the light on the paper as it passes beneath a 5150 pixel line scan camera (Sony ILX510). The view width $=50 \mathrm{~cm}$ $\times$ Number of cameras (upto a maximum of 6 meters). 
- The maximum paper web speed is 250 meters/minute. Typical web speeds are in the range of 100 to 200 meters/minute \pm 5 meters/minute. Any design solution must account for the ramp up speed of the web line.

- There is an approximate separation between watermarks of $29.7 \mathrm{~cm}$ (i.e. the length of an A4 sheet).

- Each watermark must be registered with respect to the leading edge of the paper web.

- The watermark orientation is the same as the chain lines. The direction of the movement of the paper is the same as the chain lines, although some minor skewing of the paper may occur. (Due to the dimension of the paper-web, significant skew is unlikely to occur).

- Required registration tolerance is .1 mm (approx.).

- Variables: Paper quality, texture and colour.

A key problem with web based imaging is to produce an even light profile across the web. While the imaging techniques discussed in Section 3.1 can be used to reduce a lighting gradient, it is desirable to prevent this problem from occurring. The production system uses a custom lighting unit, consisting of 
a focused backlighting strip, in conjunction with a high resolution line scan CCD camera to help minimize this gradient.

The current procedure for paper registration requires the analysis of registration marks which are placed on the outer edges of the paper web. This produces a significant amount $(\approx 3 \%)$ of waste paper as this registration region is later removed. A number of paper mills have developed in-house systems based on the use of the edge trim registration points. Results from these systems are not generally in the public domain as they would be considered commercially sensitive.

\section{Plain Paper}

Global thresholding cannot be reliably used for extracting watermarks on plain paper. This is due to both the uneven illumination function and the very low contrast of the watermark itself. Keeping in mind the speed constraints, we found that a morphological white top-hat transform followed by a double threshold and some additional cleaning filters represents the best solution.

\subsection{White Top Hat Transformation}

The choice of a given morphological filter is usually driven by the available knowledge about the shape, size, and orientation of the structures one would 
like to filter out or extract [4]. For example, we may choose an opening by a $2 \times 2$ square structuring element (SE) to remove positive impulse noise or a union of openings with line segments to extract elongated bright image structures. Morphological top-hats proceed a contrario. Indeed, the approach undertaken with top-hats consists in using knowledge about the shape characteristics that are not shared by the relevant image structures. An opening with a SE that does not fit the relevant image structures is then used to remove them from the image. These structures are recovered through the arithmetic difference between the image and its opening. This operation is called white top-hat or top-hat by opening [5].

Since the watermark consists of a network of thin lines, we have to consider an opening with a square slightly larger than the width of these lines. The opened image provides us with the illumination function which is then subtracted from the original image to enhance the watermark. This procedure is illustrated in Figure 3.

\subsection{Double threshold}

The double threshold operator DBLT thresholds the white top-hat image for two ranges of grey scale values, one being included in the other. The threshold $T$ for the narrow range is then used as a seed for the reconstruction of the 
threshold for the wide range $[6,4]$ :

$$
D B L T_{\left[t_{1} \leq t_{2} \leq t_{3} \leq t_{4}\right]}(f)=R_{T_{\left[t_{1}, t_{4}\right]}(f)}\left[T_{\left[t_{2}, t_{3}\right]}(f)\right]
$$

where $R$ denotes the morphological reconstruction by dilation of the marker image $T_{\left[t_{2}, t_{3}\right]}$ with respect to the mask image $T_{\left[t_{1}, t_{4}\right]}$. The resulting binary image is much cleaner than that obtained with a unique threshold. Moreover, the result is more stable to slight modifications of threshold values. The double threshold technique is very similar to the hysteresis threshold proposed in $[7]$.

The final filtering stage is based on a surface area criterion applied to the closing of the double threshold image: all connected components having a number of pixels smaller than a given threshold are removed. The filtered closed image is then intersected with the double threshold image. The whole procedure is illustrated in Figure 4, starting from the top-hat image shown in Figure 3c.

\section{Paper with Laid and Chain Lines}

If we perform a white top-hat and a threshold on non-plain papers, we extract the watermark and the laid/chain lines. (This is due to the fact that both the lines and the watermark are of similar intensity). The problem is to remove these lines so as to output a clean mask of the watermark. We have explored 
two different approaches: the first is based on the Fourier transform and the second uses morphological operators.

\subsection{Fourier based Segmentation}

The Two-dimensional Discrete Fourier Transform (DFT) is a powerful tool in the extraction of periodic information in digital image processing applications. Several algorithms have been developed to calculate the twodimensional DFT. The simplest makes use of the observation that this is a separable transform which can be computed as a sequence of two onedimensional transforms. Therefore, we can generate the two-dimensional transform by calculating the one-dimensional DFT along the image rows and then repeating this on the resulting image but, this time, operating on the columns. This reduces the computational overhead when compared to direct two-dimensional implementations.

Although this is still computationally slow compared to many other segmentation techniques, the Fourier transform is quite powerful. It allows the input to be represented in the frequency domain, which can be displayed as a pair of images. (It is not possible to represent both amplitude and phase using a single monochrome image.) Once the processing within the frequency domain is complete, the inverse transform can be used to generate a new image in the original spatial domain. The Fourier power, or amplitude, spec- 
trum plays an important role in image processing and analysis. This can be displayed, processed and analysed as an intensity image. Fortunately, this is not just an algorithmic transformation but a physical one [8]. The FT can also be implemented in real time using optical techniques $[9,10]$. Optical computational devices enable parallel computation of Fourier transforms, thereby presenting rapid processing.

The periodic nature of the laid and chain lines indicates the possibility of examining and filtering the paper images in the Fourier domain. To remove laid and chain lines, the filter design must contain a priori knowledge about the line properties. The images are transformed into the Fourier domain using the DFT (Figure 5). Due to their higher frequency, the laid lines appear as distinctive frequency spectrum peaks in this domain, see Figure 5(b). The lower frequency chain lines are not as obvious, since they are hidden by the significant amount of low frequency information in the image. A selective lowpass filter can be applied locally to the high frequency peaks, representing the laid lines, in the Fourier spectrum. The filter used is an ideal low-pass filter given by the filter function $H(u, v)$ :

$$
H(u, v)=\left\{\begin{array}{rr}
1 & \text { if } \sqrt{u^{2}+v^{2}} \leq \omega_{0} \\
0 & \text { otherwise }
\end{array}\right.
$$

where $\omega_{0}$ is the cut-off frequency. This filter is applied to each of the high frequency peaks representing the laid lines, see Figure 5(c). When the inverse 
FT is applied to this image we are left with a representation of the watermark without the laid lines. The white top-hat transformation (Section 3.1), thresholding and filtering (Section 3.2) stages are then applied to the resultant filtered image. Finally we apply a mask to eliminate boundary pixels. This procedure is illustrated in Figure 5. While the resultant image has been degraded, we are left with sufficient information to allow us to register the watermark on the paper sheet.

Whereas this process is efficient in removing the laid lines, the chain lines are clearly visible. Fortunately the chain lines are easily detected (i.e. by scanning the horizontal profile on a line by line basis) and can be masked out from the image. We have also examined the application of an adaptive low pass filter (based on applying a filter to the DFT power spectrum (spectral density)), but this failed to produce significantly different results, see [11] for further details.

\subsection{Morphology based segmentation}

This procedure is based on the design of a morphological filter for extracting the laid and chain lines. We perform a horizontal opening with a long line segment for extracting the laid lines (a morphological opening consists in performing an erosion followed by a dilation with the transposed SE). An opening with a vertical line segment allows us to extract the vertical chain 
lines. The union of both images is still an opening. This is a well known theorem in morphology: any point-wise maximum of a series of openings is itself an opening [12].

In our application, a morphological opening with long line segments is too sensitive to small gaps along the lines we would like to extract. An efficient solution to this problem is to consider a parametric opening which is not as 'strong' as the corresponding morphological opening. Rather than using a line structuring element $L$ of $n$ pixels, we consider all subsets of this structuring element which contains only $k$ pixels $(k<n)$. The parametric opening is then defined as the union (or point-wise maximum $\vee$ for grey scale images) of the morphological openings with these SEs:

$$
\gamma_{L_{n, k}}(f)=\bigvee_{i}\left\{\gamma_{B_{i}} \mid \operatorname{card}\left(B_{i}\right)=k \text { and } B_{i} \subseteq L_{n}\right\}
$$

where $\operatorname{card}\left(B_{i}\right)$ denotes the cardinal number (number of pixels) of $B_{i}$. Fortunately, we do not need to compute all morphological openings in parallel, as there are $\frac{n !}{k !(n-k) !}$ such openings. This is because it can be shown that the union of all these openings equals the point-wise minimum operator $\wedge$ between (i) the original image $f$ and (ii) the dilation $\delta$ with the full line segment $L_{n}$ of the rank filter of the original image using the full line segment as mask and selecting the rank $n-k+1$ :

$$
\gamma_{L_{n, k}}(f)=f \wedge \delta_{L_{n}}\left[\operatorname{Rank}_{L_{n}}^{n-k+1}(f)\right]
$$


Due to the rank operator appearing in this latter equation, this opening is also referred to as a rank-max opening $[13,14]$.

Once the parametric opening has been computed, we subtract it from the original image so as to enhance the watermark (it is therefore another white top-hat transform). We then threshold the white top-hat and perform additional cleaning filters (i.e. using the filtering process discussed in Section 3.2). The proposed steps for the morphological processing of non-plain paper are illustrated in Figure 6.

The computation load of the opening step is reduced when using the fast recursive algorithm detailed in $[15,16]$ for the morphological dilation and the moving histogram technique [17] for computing the rank filter.

\section{Real-time Watermark Registration}

While both the Fourier and the morphological approaches perform well, the morphological approach offers greater possibilities for real-time implementation. The proposed method for real-time watermark registration (see Figure 7) consists of a succession of straightforward morphological operations followed by the generation and analysis of a vertical signature. These processing steps are outlined below:

1. Capture the input region of interest. For plain paper analysis proceed to Step 3, otherwise continue. 
2. Vertical filtering stage: This additional step is only required for the analysis of the laid/chain line paper samples. During this pre-processing stage the influence of the laid lines are diminished with the help of a vertical erosion operation. (The chain lines have very little effect on the vertical profile, therefore there is no need to filter them out). Another suggested approach involves the use of opening in place of the erosion operator. This has the advantage of recovering the high values for the parts of the watermark that have been eroded. But we found that the use of erosion operator is preferable as it allows a unitary approach to both the plain and laid paper watermark analysis (i.e. the predefined values used in Step 4 will vary if we use opening).

The erosion operation was implemented using the recursive filtering algorithm developed in [16] for a range of vertical structuring element sizes varying from 3 up to 11 (depending on the magnification factor of the watermark). The final implementation, as illustrated by the results in Section 6, uses a SE of size 3. Although with a SE of this size the raw implementation of the erosion operation is just as fast as the recursive filtering algorithm. This SE provides the best compromise between the removal of the laid lines and the preservation of the vertical signature. Longer structuring elements offer an improved removal of the 
laid lines but they alter the vertical signature due to the presence of long vertical segments in the binary image obtained after the reconstruction by dilation stage (i.e. Step 5). It should be noted that the movement of the paper beneath the camera helps to blur out the effect of the laid lines and hence makes this pre-processing step much easier.

3. Top-hat: The application of the morphological top-hat operator reduces the effects of illumination gradient while preserving the thin features of the image. It also shifts the histogram back to the origin therefore simplifying the histogram analysis process in Step 4.

4. Histogram analysis: This is a key step and has a significant impact on the systems robustness. Here, two threshold values are estimated based on a histogram analysis of the image. The first threshold value $t_{1}$ is used to obtain the marker image, while the second threshold value $t_{2}$ is used to generate the mask image, where $t_{1}>t_{2}$. (See Figure 4 ). Both binary images are required in the reconstruction by dilation stage (see Section 3.2).

This histogram analysis technique is based on the following assumptions:

- The histogram is shifted back to the origin and the noise has a Gaussian distribution. 
- The average value of the watermark pixels are greater than the background pixel average.

- The number of pixels belonging to the watermark is relatively small compared to the total number of pixels of the image.

The threshold levels $t_{1}$ and $t_{2}$ are estimated by analysing both the standard and cumulative histograms. An empirical approach to the determination of the mask threshold $t_{2}$ is to allocate to it a value that is twice the grey scale value associated with the standard histogram peak. (As a result of the top-hat operation the image histogram is shifted to zero, therefore allowance is made to ensure $t_{2} \neq 0$ ). The watermark images have a strong vertical profile (i.e. the two strong peaks). This means that the threshold values $t_{1}$ and $t_{2}$ need not be determined precisely.

Let us define $N$ as the total number of white pixels in the image, $N_{t_{1}}$ as the total number of pixels with a grey level $\leq t_{1}$ (marker threshold) and $N_{t_{2}}$ as the total number of pixels with a grey level $\leq t_{2}$ (mask threshold). $N, N_{t_{1}}$ and $N_{t_{2}}$ are derived directly from the cumulative histogram, see Figure 8. Therefore the total number of white pixels in the marker image $N_{\text {marker }}=N-N_{t_{1}}$ and the total number of white pixels in the mask image $N_{\text {mask }}=N-N_{t_{2}}$. 
The marker threshold $t_{1}=t_{2}+c$ is initially applied to the image with the constant $c$ set to a predefined value. The ratio $\frac{N_{\text {marker }}}{N_{\text {mask }}}$ is then checked to ensure that we have reduced the noise sufficiently while retaining as much of the watermark as possible. If this ratio is too small then watermark image has been poorly preserved, so $t_{1}$ is decreased by decrementing $c$. If the ratio is too large, then we have too much noise in the image, therefore we increase $t_{1}$ by incrementing $c$. Based on experimentation this ratio should be in the range $.4 \leq \frac{N_{\text {marker }}}{N_{\text {mask }}} \leq .6$, these bounds being dependent on the image contrast. The gap between the higher and the lower threshold levels is usually between 3 and 5 grey levels. This method is very fast and has been successfully verified on several test images. (Out of 23 test images this approach failed only twice. In both cases the failure was due to insufficient illumination of the watermark).

Perhaps a simpler and robust threshold technique would consist of selecting the threshold level that outputs a percentage of object pixels close to the ideal watermark. This method [18] has the disadvantage of being based on the assumptions that the size of image is known $a$ priori (this is rarely the case), and that we have an ideal watermark to work from (this is difficult to estimate). Moreover, the start-up pro- 
cedure outlined in Section 5.1 works irrespectively of the presence of a watermark in the image. This would not be the case if we were to adopt the latter approach to thresholding.

5. Reconstruction by dilation: In the reconstruction by dilation operation, the mask image is recovered by successive dilations of the marker image using the fast reconstruction algorithms detailed in [19]. This technique is a preferable alternative to simple thresholding operation especially for images corrupted by noise.

6. Vertical signature analysis: This step consists in taking the vertical projection of the binary image resulting from Step 5. This process is illustrated in Figure 9. The analysis of the signature shape reveals two prominent peaks corresponding to the top and bottom co-ordinates of the watermark text. By identifying these peaks we are able to find the vertical position of the watermark within the paper sheet. An accurate estimate of the size of the watermark can also be derived from this measurement. This could also prove useful for watermark verification and classification purposes.

Another possible approach involves avoiding thresholding the image in the first place. Such an implementation can generate the signature based on the sum of the grey levels along the horizontal direction. While this approach is 
faster (it can reduce the processing time by up to $20 \mathrm{~ms}$ per watermark) and easier to implement, the signature is generally much weaker and therefore prone to generating registration errors. Since we are currently operating at real-time rates, see Section 6, this option was disregarded in the current system implementation.

\subsection{Start-up procedure}

The purpose of this procedure is to extract and register the first watermark based on a line by line examination of the CCD sensor data once the system has been initialised. When this first watermark is registered and assuming that the watermarks are evenly spaced, then we can can jump forward to the next watermark, therefore eliminating the need to examine sensor data which does not contain watermarks.

Since a simple sum of grey level pixels along the horizontal lines gives unreliable results we have adopted a method based on window analysis. This involves extracting a small window from the row image and analysing it using algorithm as presented previously. If the vertical signature does not reveal any significant peaks then we advance the window. The process continues until two prominent peaks are detected. 


\subsection{Moving window processing}

Once the first watermark is detected we can then predict the location of the next watermark, i.e. each watermark should be separated by an A4 length. This reduces the need to search each scan line for the appropriate profile and hence increases the systems speed. Occasionally situations arise where the watermarks are not evenly spaced, for example paper joins or cuts can vary this spacing. While this can be accounted for by varying the height of the moving window used during start-up procedure, it rarely causes a problem in the registration procedure.

\section{$6 \quad$ Testing and Results}

The proposed method has been tested on plain and laid/chain line paper sam-

ples with varying watermarks, see Figure 10. The images were captured by a CCD line scan camera at a typical production line rate of 120 meters/min. As the system ramps up its speed each scan line is processed to find the first watermark. This is implemented by searching for the desired vertical profile. Results are displayed in Figures 11 and 12. The double vertical lines indicate the presence of the first watermark detected. The registration point is defined as midway between these lines. The single white lines indicates the registration point for each watermark. 
The total processing time for the processing of 6 watermarks at a typical line speed of 120 meters/min and using a 60 pixel moving window, including the start-up procedure and the detection time for each watermark, is approximately 1.13 seconds when implemented on a Pentium Pro $233 \mathrm{MHz}$ (with 64 MB RAM and running NT 4.0) personal computer. The worst case start-up procedure took $661 \mathrm{~ms}$, which occurs when the first watermark is located on the lowest position of the window. This results in a processing time of $160 \mathrm{~ms} /$ watermark. Faster results can be obtained by using a smaller window. (The watermark text is the key feature within the watermark that is used to generate the vertical signature, hence our ability to reduce the window size). For example, a 40 pixel window produces a processing time of approximately $110 \mathrm{~ms} /$ watermark, well within the required time frame for real-time operation. (Real-time operation requires a processing time of 150 ms/watermark).

Figure 13 illustrates the relationship between the processing speed per watermark (excluding the start-up procedure overhead) as a function of the size of the moving window. Small window sizes are not practical as we do not have sufficient information within the window to register the watermark correctly. Although as the line speed increase the image data is compressed, thus allowing smaller processing windows. With larger window sizes the window may straddle two or more watermarks thus confusing the registration 
process. Window sizes between 40 and 60 pixels appear to offer a good compromise between the systems speed and robustness (i.e when considering the possibility of uneven distances between watermarks). The worst case for start-up procedure was assumed for the results illustrated in Figure 13.

\section{Conclusions}

Fourier and morphology based segmentation of non-plain papers have been proposed. While both approaches performed well, the morphological based segmentation produces slightly better results and is better suited to a realtime implementation on a conventional personal computer. The combination of the morphological approach and the vertical profile analysis has produced a robust real-time registration technique for both plain and chain/laid line paper samples. The system has also been shown to produce satisfactory results even when the quality of image is poor. The main benefit of this system is that it enables paper manufactures to stop producing the watermark key strips which are currently used for paper registration. These strips are added to either side of the paper and represent $\approx 3 \%$ of the total amount of paper produced on the registered grades. The data produced by the registration system can also be fed back to control the spacing between the watermarks as they are placed on the paper, hence preventing misregistration in the first place. 
While further testing of the prototype system needs to be carried out on a larger database of sample images to obtain the optimum set of parameters that will ensure the maximum performance of the segmentation process, the fundamentals of the system design have been fully developed and proven to be reliable.

\section{Acknowledgements}

We would like to thank Enterprise Ireland (Grant No: HE/98/250) and Technology Systems International Ltd. (Dublin) for their support. We would also like to thank the papers reviewers for their helpful comments.

\section{References}

[1] D. Stewart, R. Scharf, and J. Arney. Techniques for digital image capture of watermarks. Journal of Imaging Science and Technology, 39(3):261267, June 1994.

[2] P. Zamperoni. Wasserzeichenextraktion aus digitalisierten Bildern mit Methoden der digitalen Bildsignalverarbeitung. Das Papier, 43(4):133$143,1989$.

[3] C. Rauber, P. Tschudin, S. Startchik, and T. Pun. Archival and retrieval of historical watermark images. In International Conference on Image 
Processing (ICIP), pages 773-776, Lausanne, 1996. IEEE Press.

[4] P. Soille. Morphological Image Analysis. Springer-Verlag, Berlin Heidelberg, 1999.

[5] F. Meyer. Automatic screening of cytological specimens. Computer Vision, Graphics, and Image Processing, 35:356-369, 1986.

[6] C. Lantuéjoul and F. Maisonneuve. Geodesic methods in image analysis. Pattern Recognition, 17(2):177-187, 1984.

[7] J. Canny. A computational approach to edge detection. IEEE Transactions on Pattern Analysis and Machine Intelligence, 8(6):679-698, November 1986.

[8] P. Hanson and G. Manneberg. Fourier optic characterization of paper surfaces. Optical Engineering, 36(1):35-39, January 1997.

[9] H. Stark. Applications of Optical Fourier Transforms. Academic Press, 1982.

[10] P.A. Mitkas, G.A. Betzos, and L.J. Irakliotis. Optical processing paradigms for electronic computers. IEEE Computer, 31(2):45-51, Feb 1998. 
[11] P.F. Whelan and P. Soille. Watermark extraction in paper samples. In D. Vernon, editor, Proc. Optical Engineering Society of Ireland E Irish Machine Vision and Image Processing Joint Conference, pages 287-299, National University of Ireland, Maynooth, September 1998.

[12] J. Serra. Introduction to morphological filters. In J. Serra, editor, Image analysis and mathematical morphology. Volume 2: theoretical advances, chapter 5, pages 101-114. Academic Press, 1988.

[13] C. Ronse. Erosion of narrow image features by combination of local low rank and max filters. In Proc. 2nd Int. Conf. on Image Processing and its Applications, pages 77-81, London, 1986.

[14] C. Ronse and H. Heijmans. The algebraic basis of mathematical morphology ii. openings and closings. Computer Vision, Graphics, and Image Processing: Image Understanding, 54(1):74-97, 1990.

[15] M. van Herk. A fast algorithm for local minimum and maximum filters on rectangular and octogonal kernels. Pattern Recognition Letters, 13:517-521, 1992.

[16] P. Soille, E. Breen, and R. Jones. Recursive implementation of erosions and dilations along discrete lines at arbitrary angles. IEEE Transactions on Pattern Analysis and Machine Intelligence, 18(5):562-567, May 1996. 
[17] T. Huang, G. Yang, and G. Tang. A fast two-dimensional median filtering algorithm. IEEE Transactions on Acoustics, Speech and Signal Processing, 27(1):13-18, February 1979.

[18] R. Peyrard, P. Soille, J.-C. Klein, and A. Tuzikov. A dedicated hardware system for the extraction of grid patterns on stamped metal sheets. In I. Pitas, editor, Proc. of 1995 IEEE Workshop on Nonlinear Signal and Image Processing, pages 867-870, Neos Marmaras, 1995.

[19] L. Vincent. Morphological grayscale reconstruction in image analysis: Applications and efficient algorithms. IEEE Transactions on Image Processing, 2(2):176-201, 1993. 


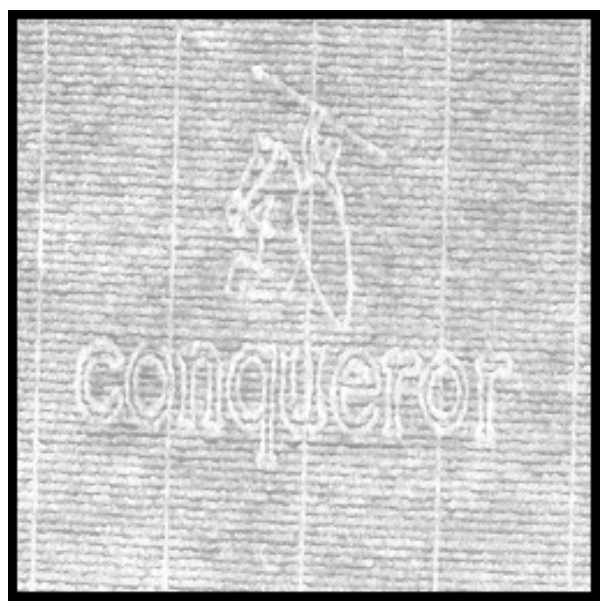

(a) Plain paper, i.e. without chain and laid

(b) Non-plain paper, i.e. with chain (vertical) lines. and laid (horizontal) lines.

Figure 1: A typical watermark on (a) plain paper and (b) paper with chain and laid lines. Note the uneven illumination on both images.

Figure 2: Production development system block diagram.

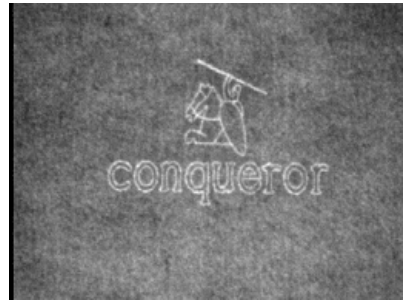

(a) Input image.

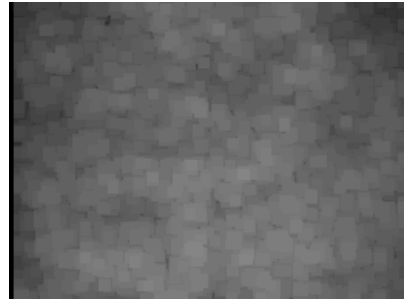

(b) Opening with a $7 \times 7$ square.

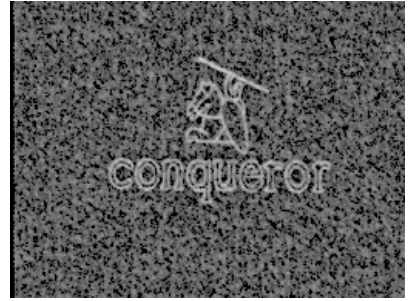

(c) White top-hat: arithmetic difference between (a) and (b).

Figure 3: From uneven to even background using the white top-hat transform. 


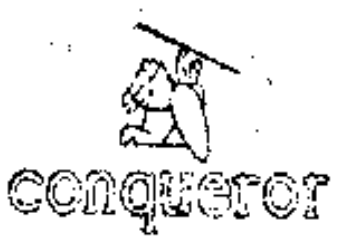

(a) First threshold of white top-hat: marker image.

$\mathbf{1}$

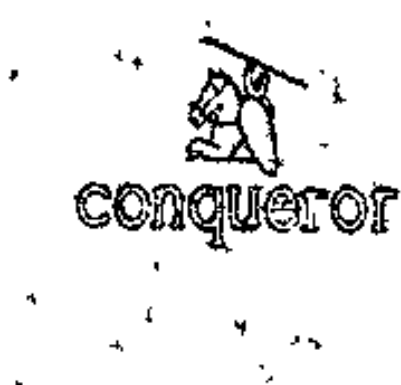

(c) Reconstruction of (b) from (a).

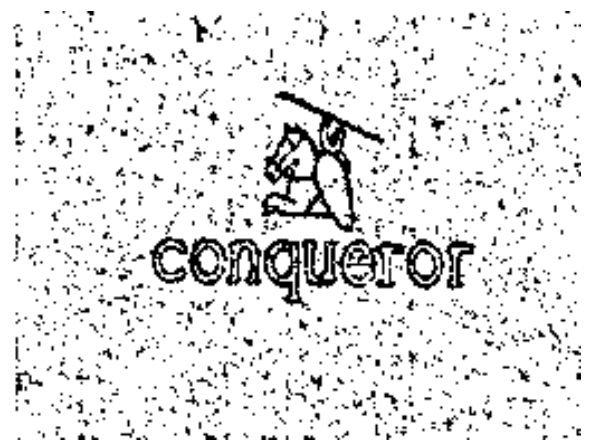

(b) Second threshold of white top-hat: mask image.

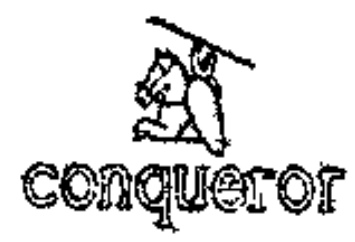

(d) After filtering based on the surface area of the connected component of the closing of (c) by a $3 \times 3 \mathrm{SE}$.

Figure 4: Extraction of the watermark from the white top-hat image shown in Figure 3c. Double threshold and additional filtering stages are also included. 


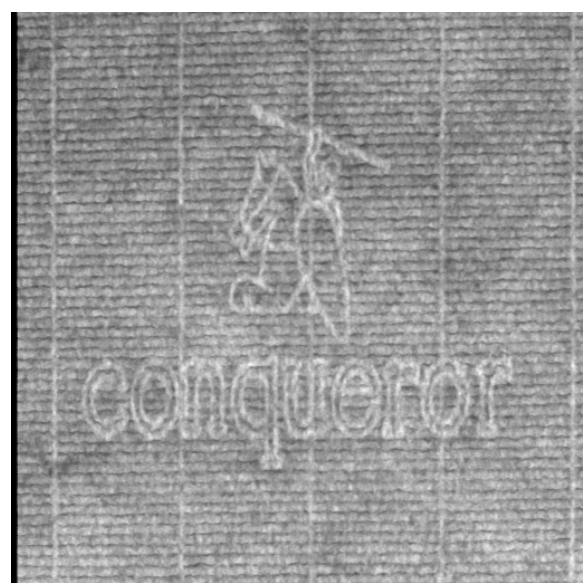

(a) Original Image.

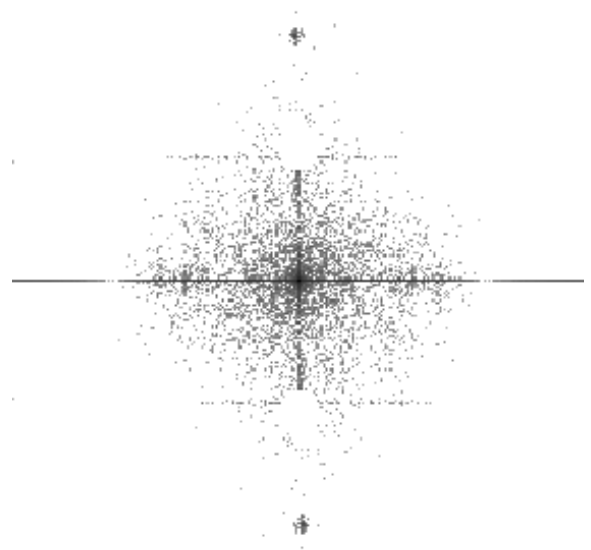

(c) Selective low-pass filtering of (b).

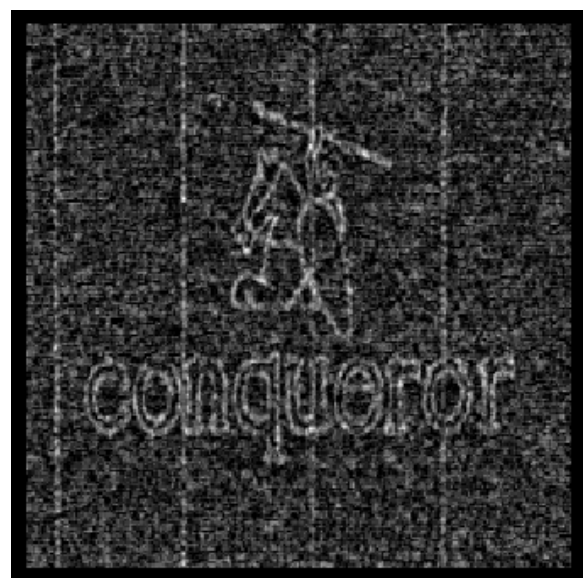

(e) White top-hat of (d).

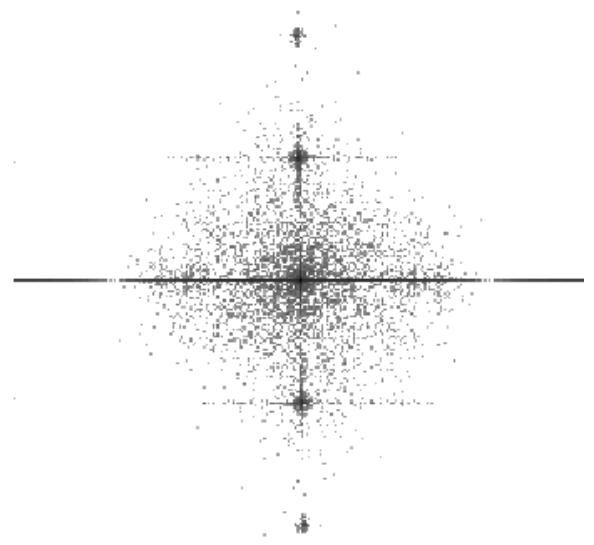

(b) Discrete Fourier Transform frequency spectrum as an intensity function.

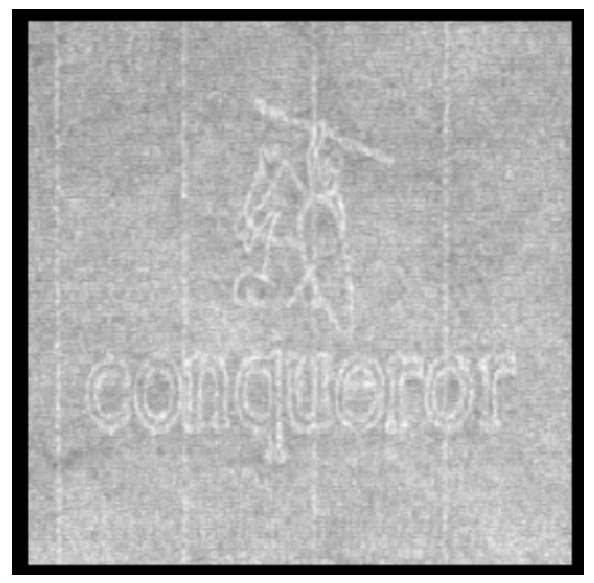

(d) Inverse Fourier Transform of (c).

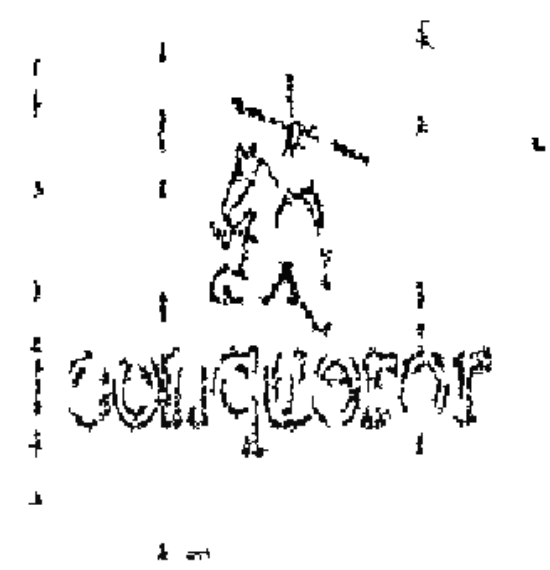

(f) Filtered double threshold of (e).

Figure 5: The application of the DFT to non-plain paper samples. 


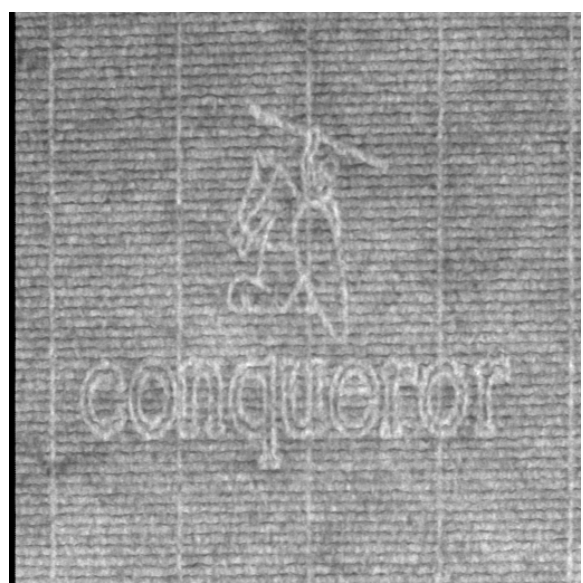

(a) Input image.

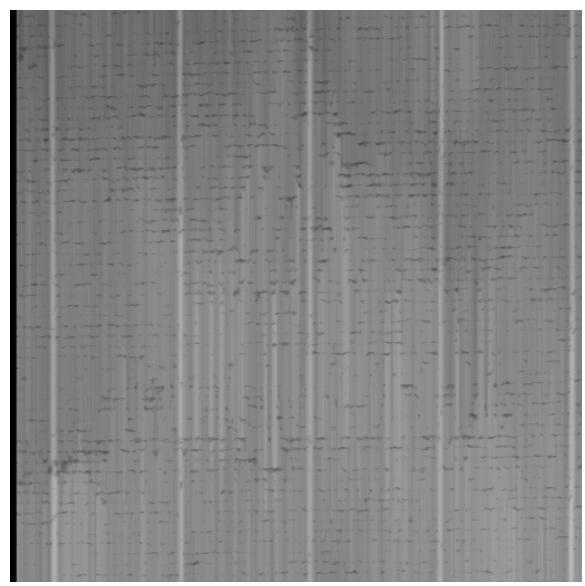

(c) Vertical parametric opening.

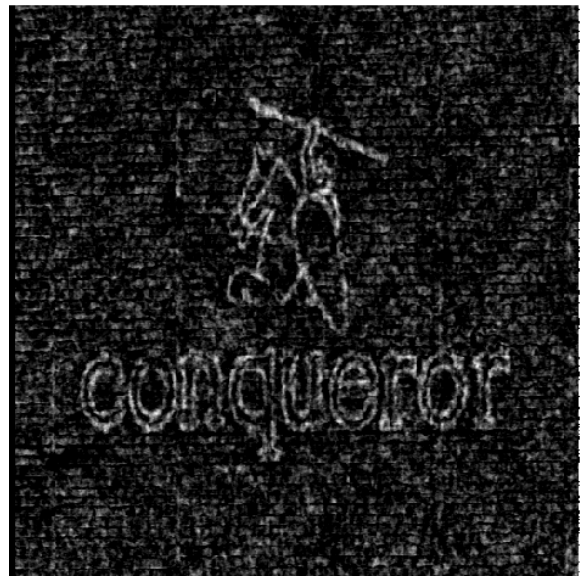

(e) Subtraction of the opening (d) from the original image (a)

Figure 6: Morphology based processing of watermarks on non-plain paper containing laid and chain lines.

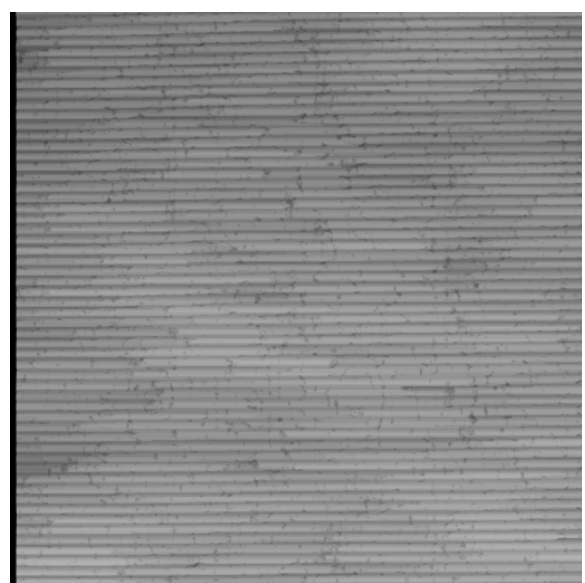

(b) Horizontal parametric opening

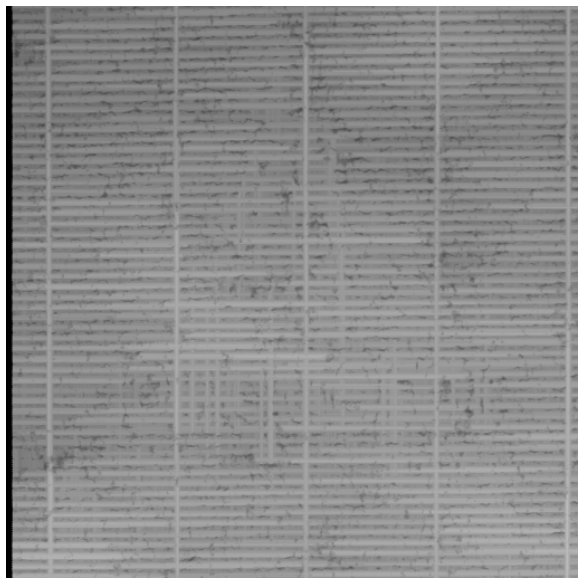

(d) Union of the two openings.

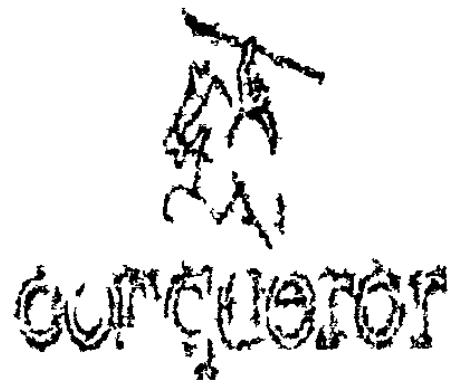

(f) Filtered double threshold of (e). 
Figure 7: Flowchart representing the real-time watermark registration process. 


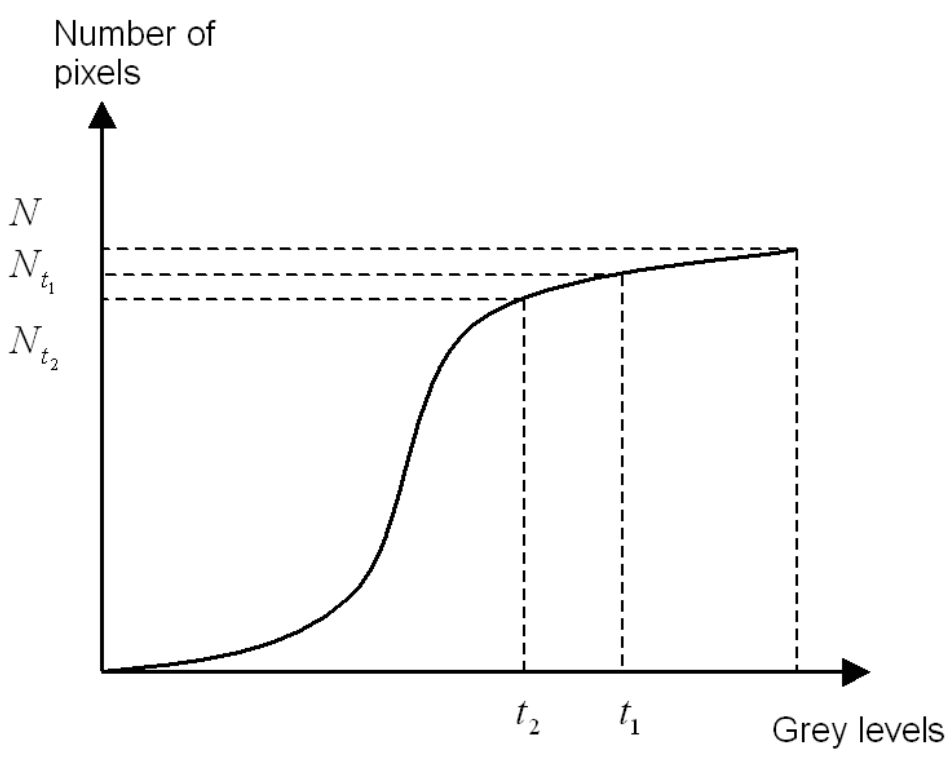

Figure 8: Using the cumulative histogram in determining the mask and marker thresholds for reconstruction by dilation.

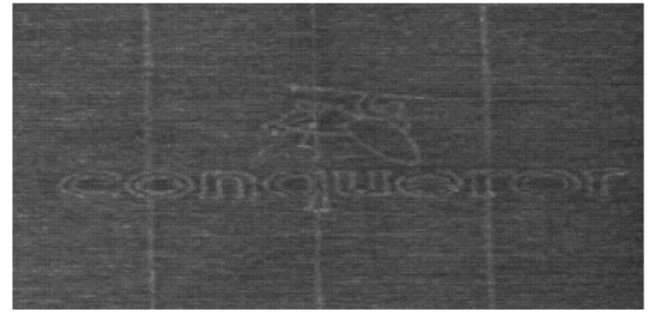

(a) Input image.

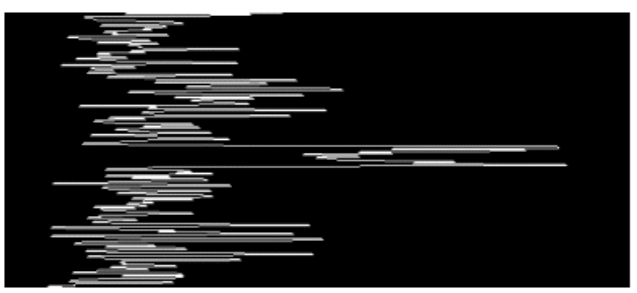

(c) Vertical signature.

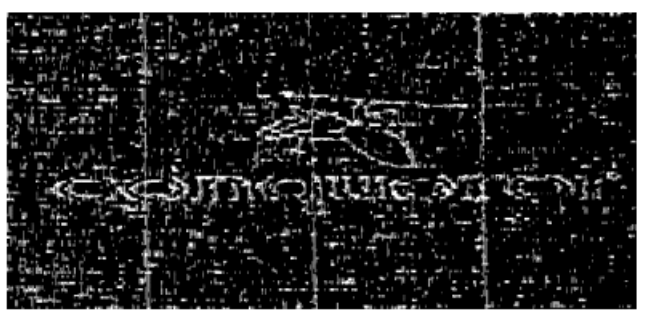

(b) Reconstruction by dilation.

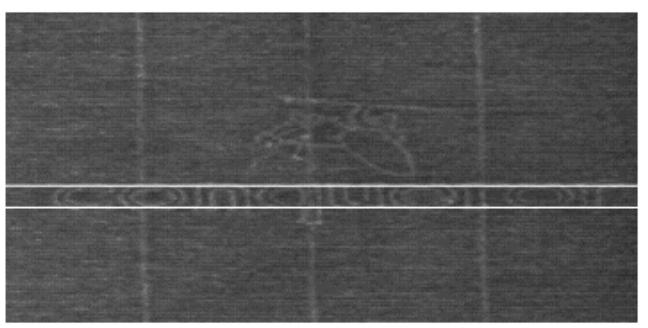

(d) The two most prominent peaks generated by the vertical signature (c) are isolated.

Figure 9: Watermark registration sequence for laid/chain line paper. 

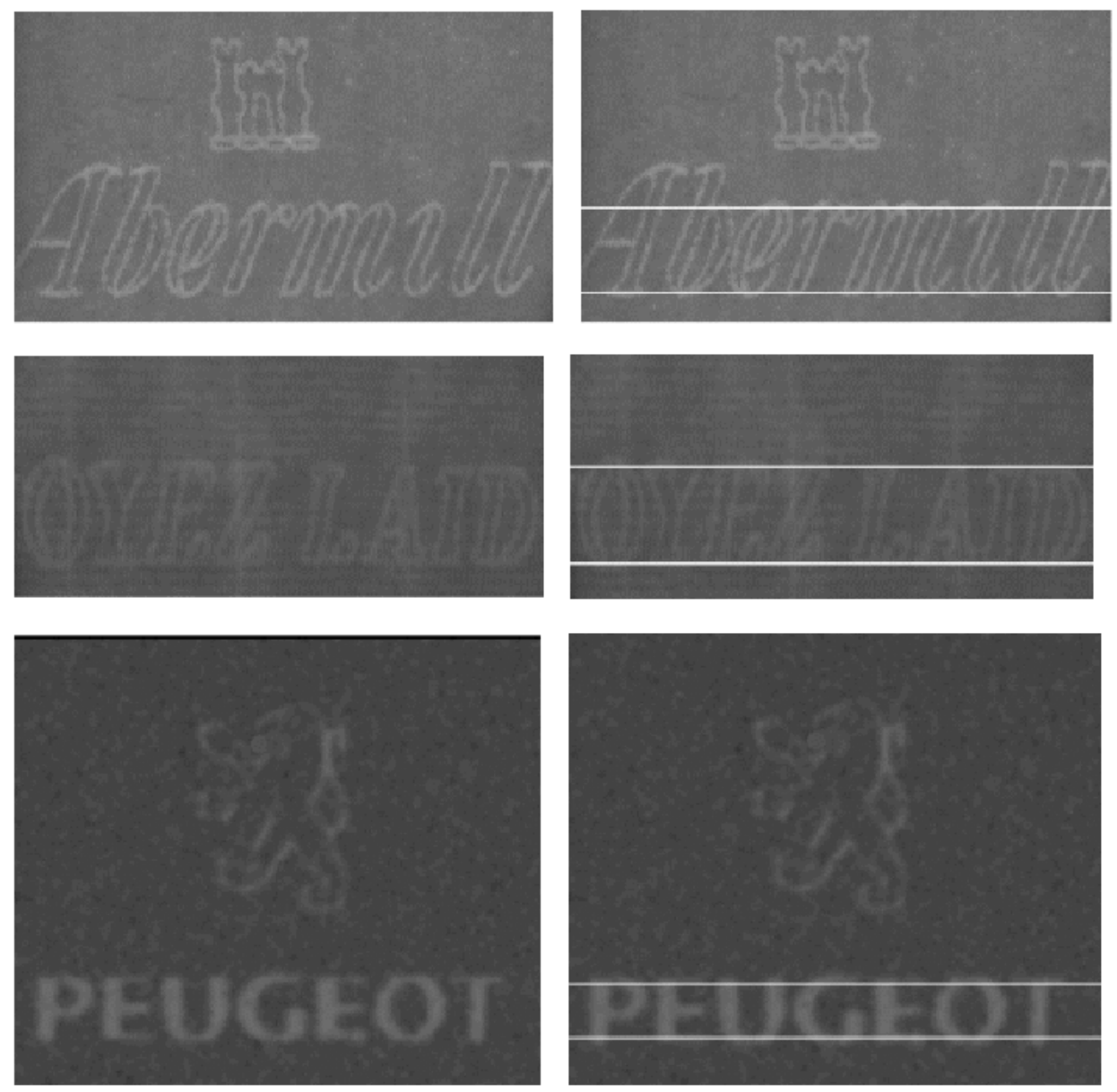

Figure 10: Application of the registration procedure to sample watermarks. 


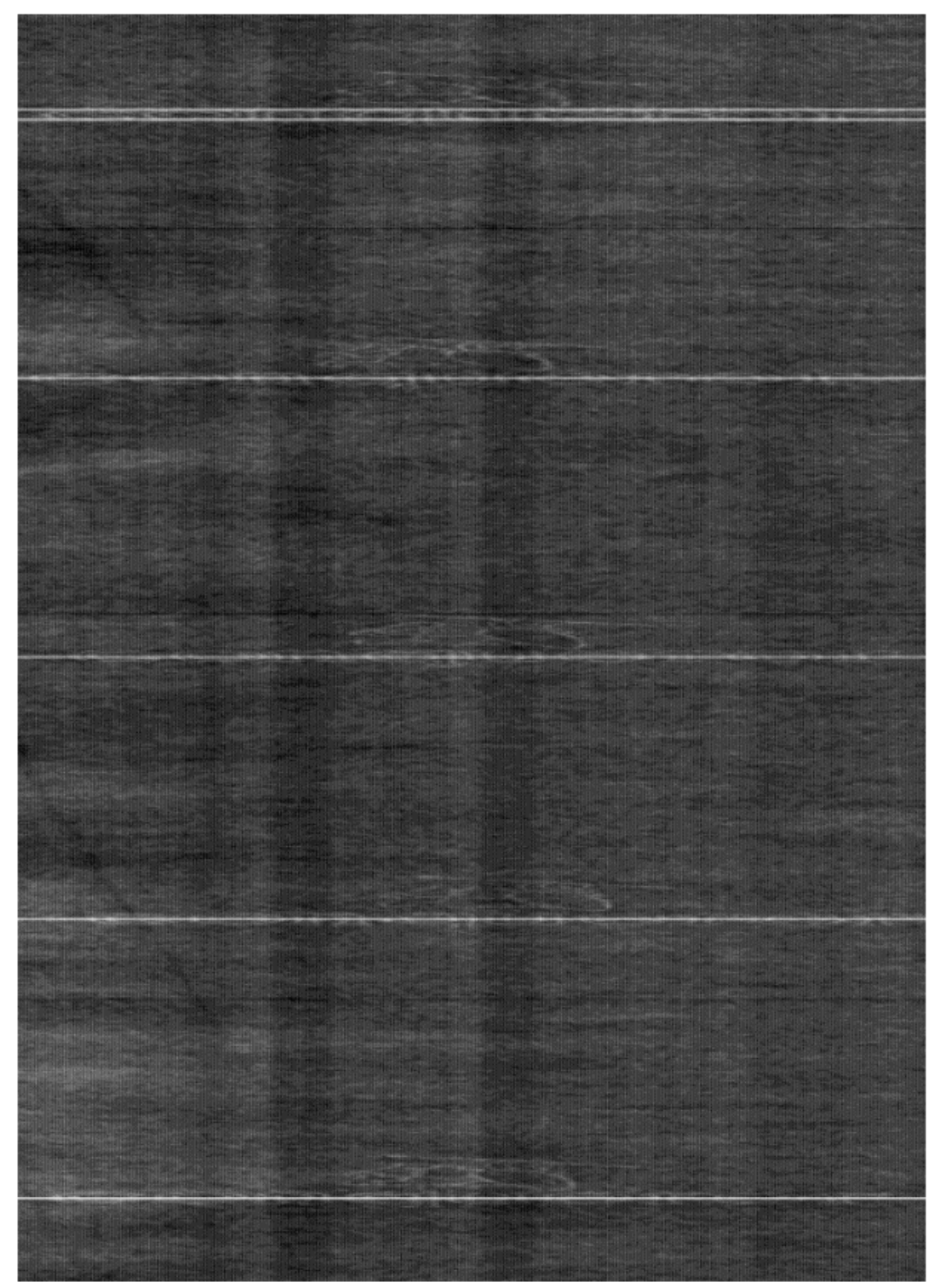

Figure 11: Real-time registration of watermarks in plain paper: Input sequence generated from a line scan camera with a paper web speed of 120 meters $/ \mathrm{min}$ and a camera resolution of .2 pixels $/ \mathrm{mm}$. The double white lines indicate the first watermark detected. Single horizontal lines indicate the detected registration point. 


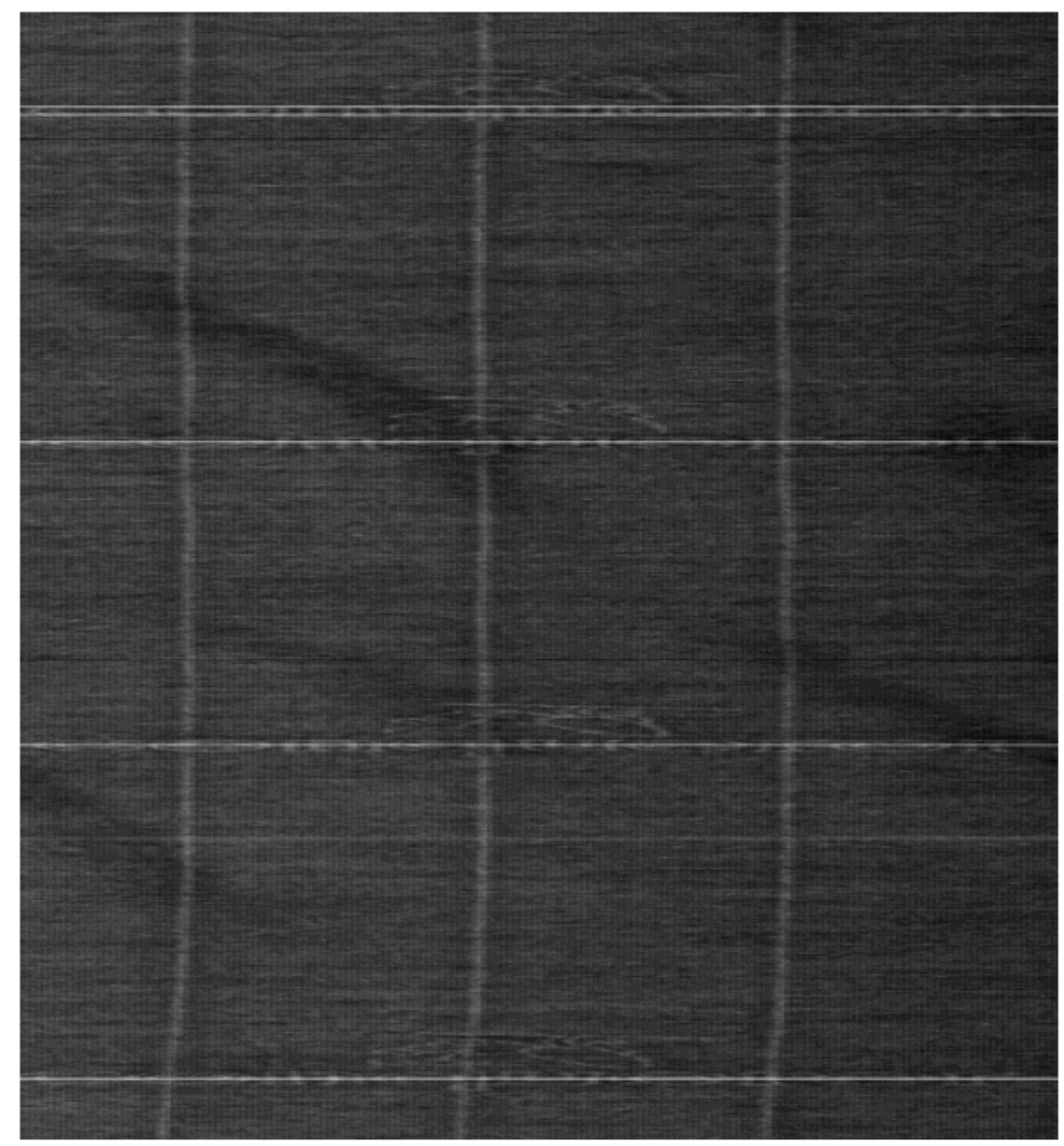

Figure 12: Real-time registration of watermarks in chain/laid line paper: Input sequence generated from a line scan camera with a paper web speed of 120 meters/min and a camera resolution of .1 pixels $/ \mathrm{mm}$. The double white lines indicate the first watermark detected. Single horizontal lines indicate the detected registration point. 


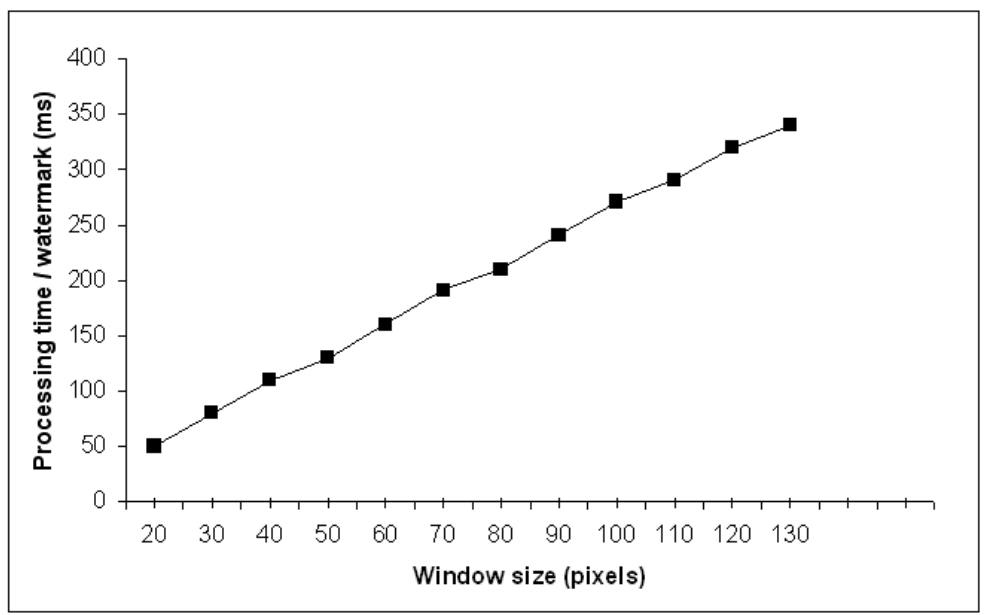

Figure 13: Processing time per watermark (excluding the start-up procedure overhead) as a function of the size of the moving window for a web speed of 120 meters/minute. At this speed window sizes less than 20 pixels are unstable as they do not contain sufficient information to register the watermark. If the window size is to big then we have the possibility the window overlapping watermarks, hence confusing the registration process. 